\title{
Vincent Di Marino, Yves Etienne and Maurice Niddam: The Amygdaloid Nuclear Complex. Anatomic Study of the Human Amygdala
}

\author{
Springer, Cham, Heidelberg, New York, Dordrecht, London, 2016, 147 p, 227 illustra- \\ tions, format $21.5 \times 28.5 \mathrm{~cm}$, ISBN: 978-3-319-23242-3, ISBN: 978-3-319-23243-0 \\ (e-Book), DOI: 10.1007/978-3-319-23243-0
}

\author{
Bruno Grignon ${ }^{1}$ \\ Received: 10 August 2016/Accepted: 11 August 2016/Published online: 16 August 2016 \\ (C) Springer-Verlag France 2016
}

Author of a magnificent Atlas of the Central Nervous System published in 2011 by Springer, Professor Vincent di Marino, aimed to provide an in-depth anatomical study of an enigmatic small gray matter structure, variably designated as a "corpus," a "complex," or a "nucleus," located in the anterior and medial part of each temporal lobe, weighing less than $1 \mathrm{~g}$ and measuring less than $20 \mathrm{~mm}$ along its longer axis, presenting multiple connections and involved in so various fields that the olfactory system, the autonomic functions, the emotional life, the sexual arousal, memory processes, and social behavior (between others): the amygdaloid complex and its inputs and outputs.

By means of dedicated dissections of half brains prepared by an injection of embalming fluid, the technique of which being carefully described, with photographs performed at each stage, sections that were treated by the $\mathrm{FeCl}_{3}$ staining technique previously used and described by the authors and providing an excellent in situ differentiation of the structures by enhancing their colors, as well as magnetic resonance (MR) images of previously dissected anatomical specimens, and MR imaging studies performed on thick sections that were secondarily dissected, and some microscopic studies devoted to the connections of particular amygdaloid areas, the authors have collected an impressive amount of original documents offering a holistic approach for studying the amygdaloid complex and its connections.

This work is composed of 12 subsequent chapters that are organized as follows: Definition and Generalities; Anatomy of Emotions via the History of Neuroanatomy and Neurosciences; Development of the Human Amygdaloid

\footnotetext{
Bruno Grignon

b.grignon@chu-nancy.fr

University of Lorraine, CHU Nancy, Nancy, France
}

Complex; Morphology of the Human Amygdala; Technique for Dissecting the Amygdaloid Body and its Close Connections; Connection Pathways of the Cerebral Amygdala; Relations of the Amygdaloid Complex; Inputs and Outputs; Vascularization of the Cerebral Amygdala; The Bed Nucleus of the Stria Terminalis; The Concept of "Extended Amygdala"; From Prefrontal Lobectomies to Amygdalectomies.

Emeritus professor of anatomy and former director of the Anatomy Laboratory at the Aix-Marseille University, Vincent Di Marino is already well known as an expert in the field of neuroanatomy with his aforementioned atlas, as well as in the field of pelvic anatomy, and as a talented teacher. Yves Etienne and Maurice Niddam, both coauthors of the Atlas of the Central Nervous System published in 2011, both former assistants of anatomy, are forensic scientists in La Timone Hospital in Marseille, France. The precious collaboration of specialists in neuroradiology, neuropathology, and research engineering has been heralded by the authors.

At a time of dramatic advances in medical imaging, particularly observed in the field of magnetic resonance imaging, including both functional MRI and MR visualization of anatomical bundles of white matter with diffusion tensor imaging and tractography, such anatomical research aiming to bridge the gap between microscopic and macroscopic observation and imaging is of paramount importance.

Richly illustrated, the book offers 227 figures of great quality, mostly composed of macroscopic views, macroscopic sections, and dissection photographs, but also including magnetic resonance images and photomicrographs illustrating embryology and histology. Well-documented, concise and easy to read, this work will be of the greatest interest to all anatomists, physicians, scientists, and researchers dealing with the central nervous system. 